\title{
ВМJ Global Health Cost-effectiveness of triage testing for facility-based systematic screening of tuberculosis among Ugandan adults
}

\author{
Matthew Murray, ${ }^{1}$ Adithya Cattamanchi, ${ }^{2}$ Claudia Denkinger, ${ }^{3}$ Anja van't Hoog, ${ }^{4}$ \\ Madhukar Pai, ${ }^{5}$ David Dowdy ${ }^{1}$
}

To cite: Murray M,

Cattamanchi A, Denkinger C, et al. Cost-effectiveness of triage testing for facilitybased systematic screening of tuberculosis among Ugandan adults. BMJ Global Health 2016:1:e000064. doi:10.1136/bmjgh-2016000064

Received 18 April 2016 Revised 12 July 2016 Accepted 26 July 2016

\section{CrossMark}

${ }^{1}$ Department of

Epidemiology, Johns Hopkins Bloomberg School of Public Health, Baltimore, Maryland, USA

${ }^{2}$ Division of Pulmonary and Critical Care Medicine, Department of Medicine San Francisco General Hospital, University of California, San Francisco, California, USA

${ }^{3}$ Foundation for Innovative New Diagnostics (FIND), Geneva, Switzerland ${ }^{4}$ Amsterdam Institute for Global Health and Development (AIGHD), Amsterdam, The Netherlands ${ }^{5}$ Department of Epidemiology \& Biostatistics, McGill International TB Centre, McGill University, Montreal, Quebec, Canada

Correspondence to Dr David W Dowdy; ddowdy1@jhmi.edu

\section{ABSTRACT}

Background: Systematic screening is often proposed as a way to improve case finding for tuberculosis (TB), but the cost-effectiveness of specific strategies for systematic screening remains poorly studied.

Methods: We constructed a Markov-based decision analytic model to analyse the cost-effectiveness of triage testing for TB in Uganda, compared against passive case detection with Xpert MTB/RIF. We assumed a triage algorithm whereby all adults presenting to healthcare centres would be screened for cough, and those with cough of at least 2 weeks would receive the triage test, with positive triage results confirmed by Xpert MTB/RIF. We adopted the perspective of the TB control sector, using a primary outcome of the cost per year of life gained (YLG) over a lifetime time horizon.

Results: Systematic screening in a population with a $5 \%$ underlying prevalence of TB was estimated to cost US\$610 per YLG (95\% uncertainty range US\$200US\$1859) with chest X-ray (CXR) (US\$5 per test, specificity 0.67), or US\$588 (US\$221-US\$1746) with $C$ reactive protein (CRP) (US\$3 per test, specificity 0.59 ). In addition to the cost and specificity of the triage test, cost-effectiveness was most sensitive to the underlying prevalence of TB, monthly risk of mortality in people with untreated TB and the proportion of patients with TB who would be treated in the absence of systematic screening.

Conclusions: To optimise the cost-effectiveness of facility-based systematic screening of TB with a triage test, it must be carried out in a high-risk population, or use triage tests that are cheaper or more specific than CXR or CRP.

\section{INTRODUCTION}

Tuberculosis (TB) is the leading single-agent cause of infectious mortality worldwide, accounting for an estimated 1.5 million deaths in 2014. ${ }^{1}$ The WHO and Stop TB Partnership have set ambitious milestones for ending TB, including a $20 \%$ reduction in incidence and $35 \%$ reduction in mortality by 2020. To achieve these milestones, novel tools and approaches for finding $\mathrm{TB}$ cases

\section{Key questions}

What is already known about this topic?

- A key challenge for reducing tuberculosis (TB) mortality lies in detecting the one-third of patients missed by passive case detection. Systematic screening has shown promise to help close this gap.

- Previous studies have not considered the costeffectiveness of scaling up TB case finding by using systematic screening in healthcare facilities.

What are the new findings?

- This model shows that using chest X-ray (CXR) and $C$ reactive protein (CRP) in this way is costeffective at US\$610 and US\$588 per year of life gained (YLG), respectively.

- Scale-up should be done in high-burden settings, or use triage tools cheaper or more specific than CXR and CRP.

\section{Recommendations for policy}

- To achieve the WHO goal of a $35 \%$ reduction in TB mortality by 2020 , substantial improvements in diagnosis must be realised. This analysis suggests that using triage tools in a clinical setting may help achieve this in a cost-effective manner.

will be essential. ${ }^{2}{ }^{3}$ One key priority in this effort is to diagnose and treat the one-third of all patients with TB who are missed every year by passive case detection. ${ }^{45}$

Systematic screening has been advanced as one way to reach this sizeable undetected population. ${ }^{6}$ The potential benefits of systematic screening include an increased diagnostic yield, shortened time to diagnosis, cost-effective return on investment and reductions in transmission. ${ }^{7-11}$ Many methods exist for systematic screening; ${ }^{12}$ one that has received increasing attention recently is facility-based $\mathrm{TB}$ screening, in which all patients presenting to healthcare facilities are actively assessed for TB symptoms regardless of the reason for 
presentation, and referred for TB testing if symptomatic. However, diagnostic tests such as sputum smear microscopy are poorly sensitive for $\mathrm{TB}$, especially in the context of systematic screening, and more sensitive diagnostic tests (eg, Xpert MTB/RIF, Cepheid, Sunnyvale, California, USA; 'Xpert') are too costly to perform on every patient with symptoms. ${ }^{13}$

One method to improve the cost-effectiveness of facility-based screening for TB is to use a 'triage' test. ${ }^{14}$ Triage tests are rapid, high-sensitivity, lower cost tools used to screen individuals before applying a more costly confirmatory test such as Xpert. ${ }^{7} 1516$ The most widely available potential triage test is chest X-ray (CXR), but other biomarker-based triage tests are being developed. $\mathrm{C}$ reactive protein (CRP) is a non-specific inflammatory marker that can be measured in minutes from capillary blood and has been suggested in early studies as a TB triage test. ${ }^{17} 18$ We developed a Markov-based decision analytic model to evaluate the cost-effectiveness of triage testing using CXR or CRP as a means to facilitate facilitybased systematic screening for active TB in Uganda.

\section{METHODS}

\section{Study setting}

Uganda is a high-burden country with an estimated TB prevalence of 154 per 100000 , HIV prevalence of $48 \%$ among people with incident $\mathrm{TB}$, and prevalence of multidrug-resistant (MDR) TB of $1.4 \%$ among new TB cases. ${ }^{1}$ For this analysis, we considered an upper-level primary health centre (health centre IV) or district-level hospital, in which Xpert testing and chest radiography is available on-site. Although not all health centres and district hospitals have this capacity, triage testing for $\mathrm{TB}$ would most likely be implemented first in centres that do. We assumed that initial screening for cough of at least 2 weeks could be performed of individuals presenting to the health centre for any reason, using a twoquestion screen and at negligible cost. Many algorithms could be implemented to identify people eligible for this initial screening; we did not attempt to specify a particular algorithm, but rather simply assumed that a population of individuals could be identified through facility-based screening for cough, and that this population of individuals with cough would have a given prevalence of TB, which we varied in sensitivity analysis. Our study population therefore consisted of all adults found to have a cough on this initial screen. This population is assumed to include the subset of individuals who, if fully evaluated, would raise sufficient clinical suspicion of active TB that an Xpert test would be ordered under passive case detection, even in the absence of cough screening or triage testing.

\section{Outcomes and economic methods}

The primary outcome of this model was the incremental cost-effectiveness ratio (ICER), reported as the incremental cost per year of life gained (YLG), comparing systematic screening with a triage test to passive case finding using Xpert for diagnosis. We adopted the perspective of the TB control sector, using a lifetime time horizon and focusing on costs incurred by the TB control programme. Thus, costs of ongoing antiretroviral therapy (ART) in HIV-infected TB survivors were not incorporated. Estimates of costs and outcomes were drawn from the published literature. Since for TB, YLGs often approximate disability-adjusted life years (DALYs) averted, ${ }^{19}$ and the cost per YLG is a conservative estimate of the cost per DALY, we used a cost per YLG of less than the per capita gross national income as a threshold for a highly cost-effective intervention. ${ }^{20}$ All costs were inflated from historical to 2014 US dollars using the US Consumer Price Index. ${ }^{21}{ }^{22}$ Capital costs were annualised over the estimated useful life of all capital equipment, and future YLGs and costs were discounted at $3 \%$ per year.

\section{Model structure}

We developed a Markov-based decision analytic model to simulate triage testing as a strategy for systematic TB screening in Uganda (figure 1). In the baseline scenario (without triage testing), we assumed that only those patients with symptoms sufficient to raise clinical suspicion of active TB would be referred for TB testing, which we assumed would be performed with Xpert MTB/RIF regardless of HIV status. We compared this to a screening scenario in which all individuals presenting to the facility for any reason would be screened for cough of at least 2 weeks, those screening positive would have a triage test performed and those with a positive triage test would be referred for Xpert. In the screening scenario, we assumed that those with sufficient clinical suspicion of active TB would receive Xpert testing, even if the cough screen or triage test result were negative. Thus, systematic screening could increase the number of individuals diagnosed and treated for $\mathrm{TB}$ relative to the baseline scenario, but could not decrease that number. Although testing using Xpert for all individuals with TB symptoms is not currently the standard of care in many Ugandan facilities, it is a globally recommended diagnostic algorithm that is practised in other high-burden settings (eg, South Africa). Furthermore, since Xpert should be implemented for people with clinical suspicion of TB before being used for systematic screening, we considered that systematic screening with a triage test would be prioritised in settings where Xpert was already being universally performed for passive TB diagnosis.

In this model, individuals with underlying active TB whose diagnosis is missed on initial presentation (either because no TB testing is performed, or because the test result is false-negative and empiric treatment is not initiated) enter a Markov loop with a time step of 1 month. At each month, the individual is assigned a probability of spontaneous resolution, death and returning to the health facility. ${ }^{23}$ Those who return to the facility enter a similar decision algorithm (screening for 


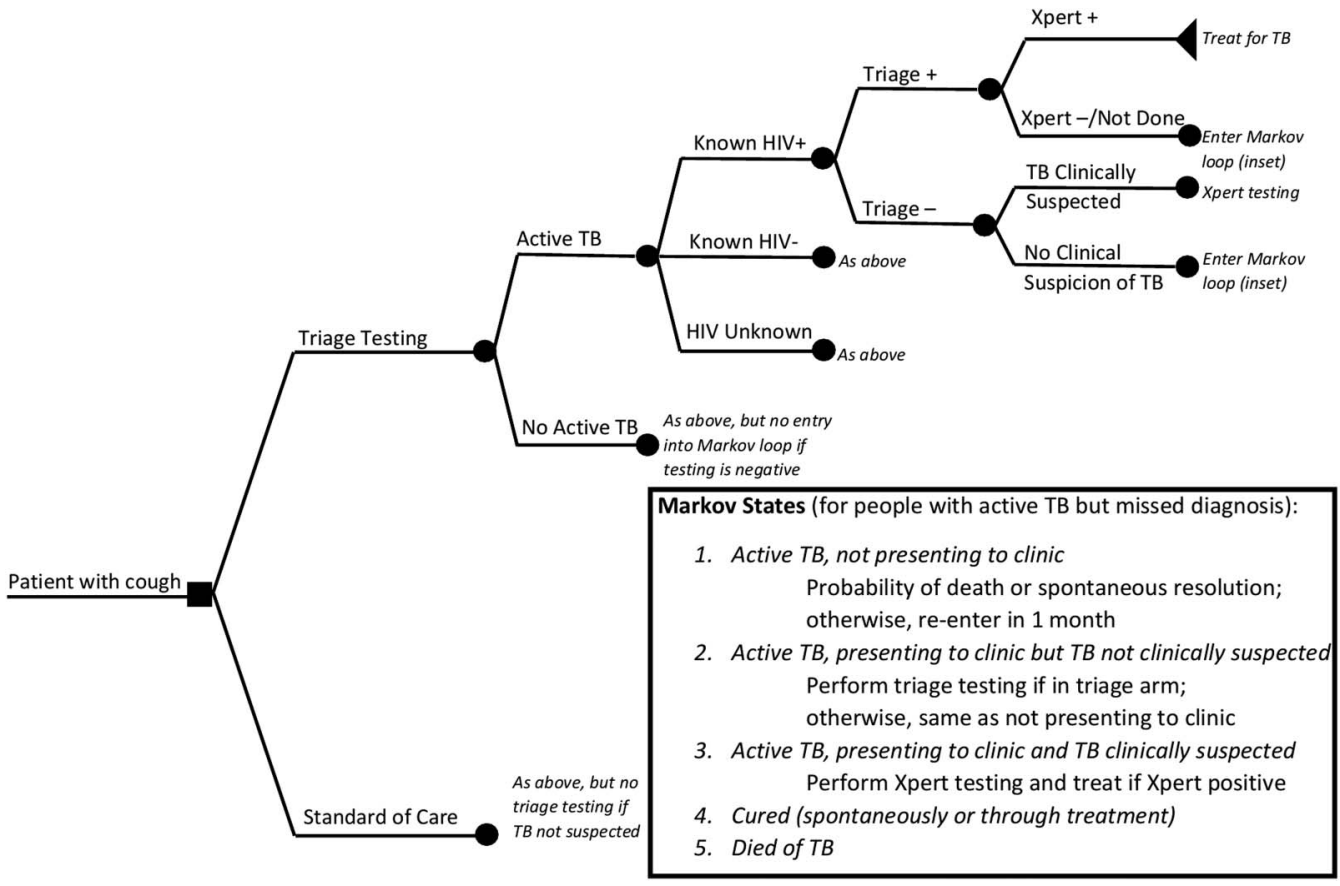

Figure 1 Model diagram. All patients with a cough of at least 2 weeks' duration are first characterised according to active tuberculosis (TB) and HIV status. In the triage testing scenario (upper branch), individuals are then tested with a triage test, with those testing positive on the triage being sent for diagnostic testing with Xpert MTB/RIF; in the standard of care (lower branch), these individuals receive diagnostic testing according to clinical judgement only. Patients with underlying active TB whose diagnosis is missed enter a Markov loop with a 1 month time step, with states as described in the inset.

cough followed by triage testing in the triage scenario, Xpert testing if active TB is clinically suspected in either scenario), with the conservative assumptions that anyone previously suspected of having active TB will be tested for $\mathrm{TB}$ on repeat presentation as well, and that Xpert testing is always positive on a repeat test in someone with active TB. For simplicity, we also assume that all deaths occur instantaneously, without accrual of any YLGs. These assumptions are relaxed in sensitivity analysis. The Markov loop is continued for all individuals with active TB until resolution (spontaneous or through treatment) or death. The model includes the possibilities of empiric treatment in the case of a negative Xpert result (whether true-negative or false-negative), losses to follow-up between ordering of Xpert and initiation of treatment, and losses to follow-up and failures after initiating treatment. Full model parameters are shown in table 1.

\section{Sensitivity analysis}

We performed one-way sensitivity analyses on all model parameters and multiway sensitivity analyses on those parameters to which model results were most sensitive. Ranges for sensitivity analysis were taken from published literature when available; where not available, wide ranges were assumed. We also performed a probabilistic uncertainty analysis by varying all parameters simultaneously across uniform distributions bounded by the corresponding ranges of all model parameters. We report $95 \%$ uncertainty ranges (URs) as the 2.5 th and 97.5 th percentiles of 10000 model simulations and use cost-effectiveness acceptability curves to describe the proportion of simulations under which each triage test would be cost-effective, as a function of willingness to pay for one YLG.

\section{RESULTS}

In the reference scenario, we assumed that $5 \%$ of all patients presenting to a healthcare facility with a cough of at least 2 weeks would have TB; thus, for every 10000 such patients, 500 would have active TB. In the absence of cough screening and triage testing, 166 were projected to be diagnosed and treated on their first presentation (10 of whom died), 285 were diagnosed and treated on subsequent presentation (17 of whom died), 4 resolved spontaneously without treatment, and 18 died of TB without ever being treated. With screening and triage testing (using CXR or CRP), 416 of these 500 patients were diagnosed and treated on initial presentation (27 of whom died), 45 on subsequent presentation (3 of whom died), and 9 were projected to die without treatment. Relative to no triage testing, screening followed by triage with CXR (US\$5 per test, specificity 0.67) was estimated to cost US\$610 (95\% UR US\$200-US\$1859) per YLG (table 2). This was similar to triage with CRP (US\$3 per test, specificity 0.59): US\$588 per YLG (US\$221-US $\$ 1746)$. Including a fully loaded annual ART cost of US\$305 (and assuming lifelong ART for all HIV-positive survivors), the incremental cost-effectiveness of CXR triage rose to US $\$ 746$ per YLG. ${ }^{33}$ On probabilistic uncertainty analysis, the probability that screening and triage testing would cost less than the per capita gross national 
Table 1 Model inputs: cohort probabilities, diagnostic parameters and costs

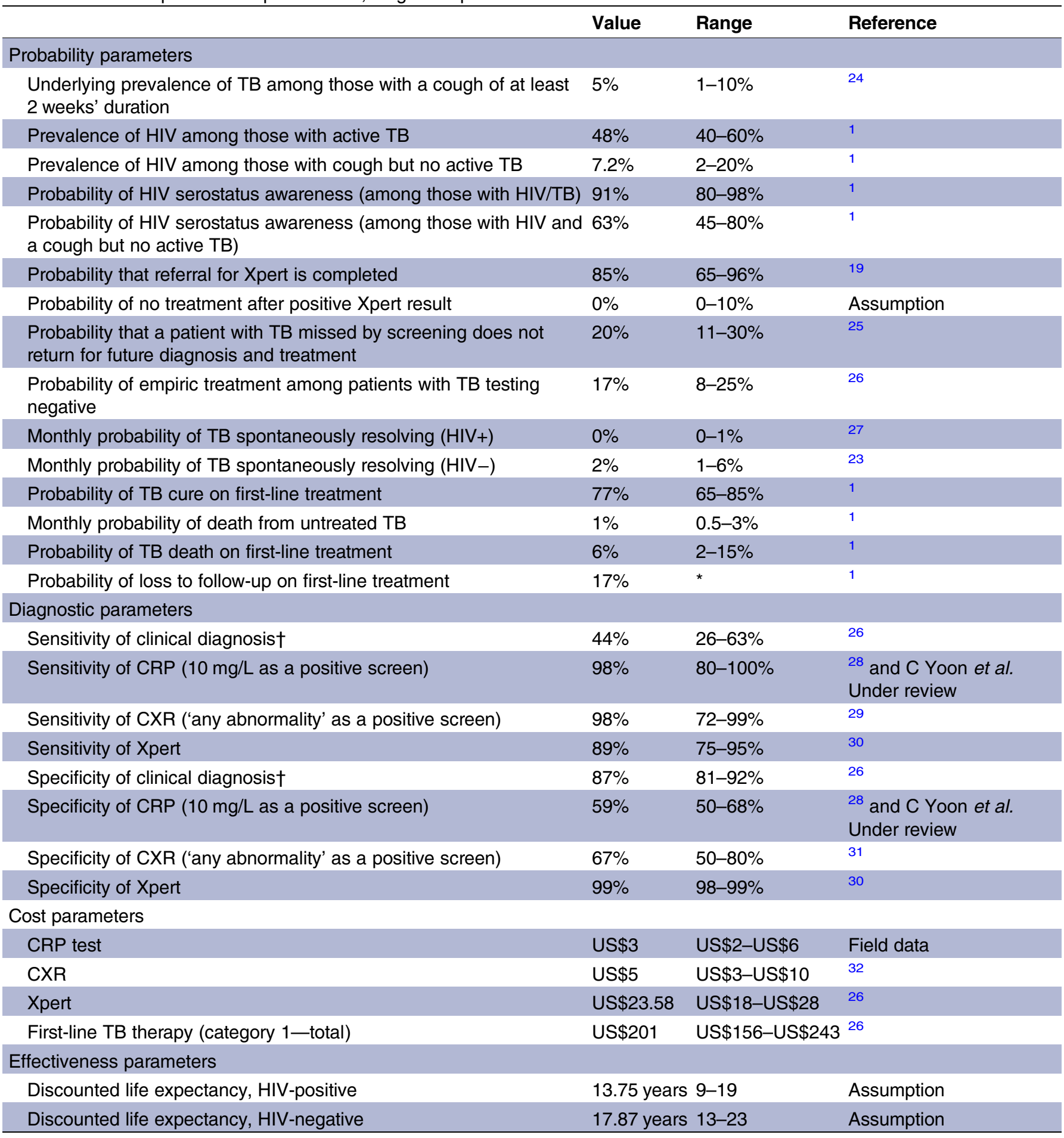

*Set equal to (1-probability of cure-probability of death).

†Probability that an individual with active TB will be referred for Xpert testing in the absence of a triage test.

CRP, C reactive protein; CXR, chest $X$-ray; TB, tuberculosis.

income of Uganda (US\$680) per YLG was 0.57 with CXR and 0.59 with CRP (figure 2).

On one-way sensitivity analysis, the parameters to which the model was most sensitive included the prevalence of active TB among the patient population with a cough; the monthly probability of death (and alternatively of spontaneous resolution) among patients with active, untreated TB; the proportion of patients with $\mathrm{TB}$ who would be diagnosed and treated in the absence of triage testing; and the cost and specificity of the triage test (figure 3).

Figure 4 shows the settings under which triage testing would be preferred to the standard of care, at a specificity of 0.67 and willingness to pay of US\$680 per YLG. If 
Table 2 Expected costs, days of life gained, and incremental cost-effectiveness of screening for cough followed by triage testing for tuberculosis, per patient presenting with prolonged cough to a healthcare facility in Uganda

\begin{tabular}{llcllll}
\hline Strategy & $\begin{array}{l}\text { Average cost } \\
\text { (2014 US\$) }\end{array}$ & $\begin{array}{l}\text { Incremental cost } \\
\text { (2014 US } \$)\end{array}$ & $\begin{array}{l}\text { Average years } \\
\text { of life lived }\end{array}$ & $\begin{array}{l}\text { Incremental days of ICER } \\
\text { life gained }\end{array}$ & $\begin{array}{l}\text { (US\$/YLG) } \\
\text { Uncertainty range }\end{array}$ \\
\hline $\begin{array}{l}\text { Standard of } \\
\text { care }\end{array}$ & US $\$ 18.23$ & - & 17.417 & - & - & - \\
CRP & US $\$ 24.30$ & US $\$ 6.07$ & 17.427 & 3.7 days & US $\$ 588$ & US $\$ 221-U S \$ 1746$ \\
CXR & US $\$ 24.52$ & US $\$ 6.29$ & 17.427 & 3.7 days & US $\$ 610$ & US $\$ 200-U S \$ 1859$ \\
\hline
\end{tabular}

Incremental effectiveness and cost-effectiveness for both CRP and CXR are presented relative to the standard of care scenario.

CRP, C reactive protein; CXR, chest X-ray; ICER, incremental cost-effectiveness ratio; YLG, year of life gained.

the price of the triage test could be lowered to US $\$ 1$ or the monthly probability of death from untreated TB was as high as 0.03 , triage testing was almost universally preferred. By contrast, in a setting where the prevalence of underlying TB among those screened was $2 \%$ or less, triage testing was generally not cost-effective at this willingness-to-pay threshold unless the per-test price could be lowered.

\section{DISCUSSION}

This Markov-based decision model illustrates the conditions under which facility-based screening for cough followed by triage testing for TB is likely to be cost-effective in Uganda. Specifically, for a triage test with high sensitivity $(98 \%)$ to be cost-effective at a willingness to pay of US $\$ 680$ per YLG in a population where the underlying TB prevalence is $5 \%$ and the monthly risk of TB death is $1 \%$, it must cost US\$3 (specificity $59 \%$ ) to US $\$ 5$ (specificity $67 \%$ ) per test. If the probability of active TB, TB death or missed TB diagnosis under the standard of care increases, then the corresponding price threshold for the triage test increases accordingly. These results suggest that, for triage testing to be cost-effective in a setting like Uganda, either currently available tests must be implemented in populations with high underlying

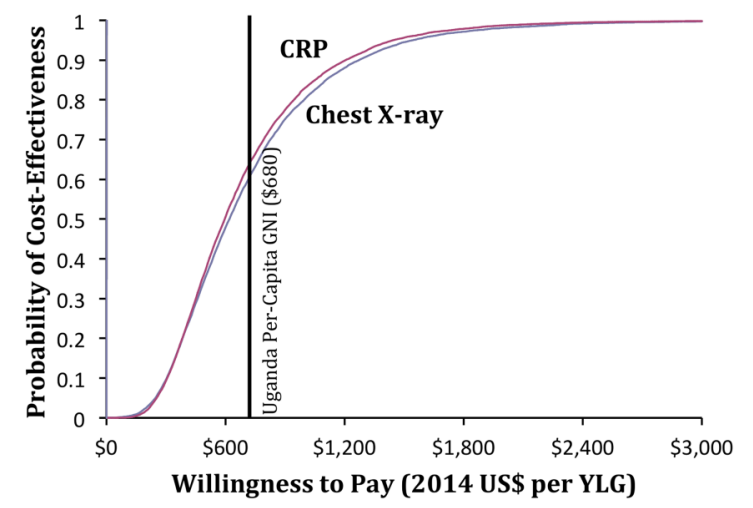

Figure 2 Cost-effectiveness acceptability curve for tuberculosis triage testing. The curve shows the probability of cost-effectiveness of triage testing with chest X-ray (CXR, blue) or $\mathrm{C}$ reactive protein (CRP, red) over 10000 Monte Carlo simulations. The vertical black line indicates the per capita gross national income of Uganda. YLG, year of life gained.
TB prevalence and high risk of death from untreated $\mathrm{TB}$, or else tests with lower cost and/or higher specificity must be developed and validated.

Our results indicate that implementing existing triage tests may be less preferred, from a cost-effectiveness standpoint, than other interventions such as scale-up of Xpert MTB/RIF for passive diagnosis of TB-which had a more favourable cost-effectiveness profile in Uganda. ${ }^{33}$ However, the unit cost of triage testing (US\$3-US\$5) currently remains somewhat high. While CXR and CRP both meet many of the standards laid out in a recently developed target product profile for triage TB tests, that profile listed US\$2 as the maximum acceptable unit cost. ${ }^{15}$ Comparison of CXR and CRP also demonstrates the inherent trade-off between lower cost and higher specificity (which saves money by reducing false-positive screening results). These findings provide strong support for ongoing development and validation of triage tests that can be performed on accessible clinical specimens (eg, capillary blood or urine) at lower cost and/or higher accuracy.

Our sensitivity analysis highlights the importance of the existing standard of care in determining the incremental cost-effectiveness of triage testing for TB. Specifically, triage testing is likely to be most cost-effective where the majority of individuals with TB will not be diagnosed otherwise on clinical grounds (figure 3, red bar for 'sensitivity of clinical diagnosis'). The cost-effectiveness of triage testing also depends on a screening algorithm that can identify a population at high risk of TB. For example, screening for cough of any duration may identify more individuals with TB than screening for prolonged cough; however, if only $1-2 \%$ of individuals with a cough of any duration have $\mathrm{TB}$, then as shown in figure 4, triage-based screening of that population with existing tests is unlikely to be cost-effective.

A previous analysis suggested a substantial reduction in diagnostic costs in Uganda for a theoretical triage test that is $90 \%$ sensitive, $75 \%$ specific and with a cost of US $\$ 5 .{ }^{34}$ Another recent study similarly suggested that CXR as a triage for Xpert testing in resource-constrained settings could increase throughput while missing few cases of active TB and reducing diagnostic costs. ${ }^{35}$ Both of these studies conceptualised triage testing as a way to reduce diagnostic costs, relative to a baseline in which Xpert testing was performed on all individuals. Our 


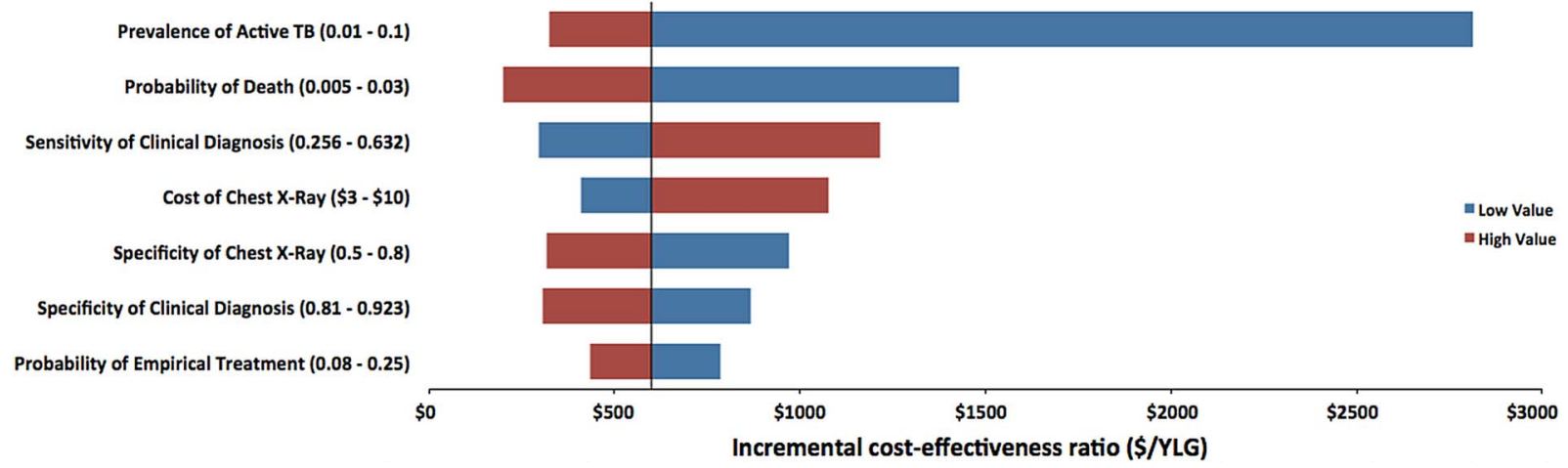

Figure 3 One-way sensitivity analysis on cost-effectiveness of triage testing for tuberculosis (TB) in Uganda. The vertical line represents the base case for chest X-ray (US\$610 per year of life gained (YLG)). Ranges for sensitivity analysis are indicated alongside the seven most influential parameters.

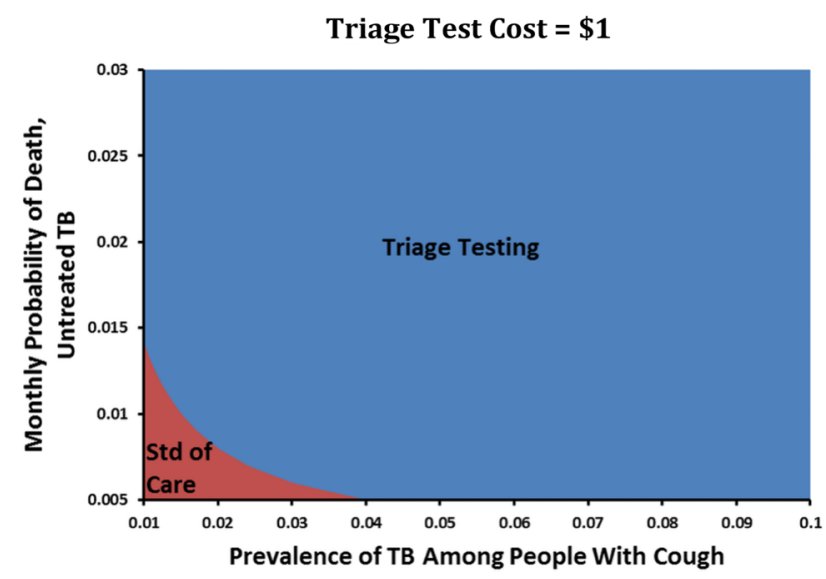

Triage Test Cost $=\$ 5$

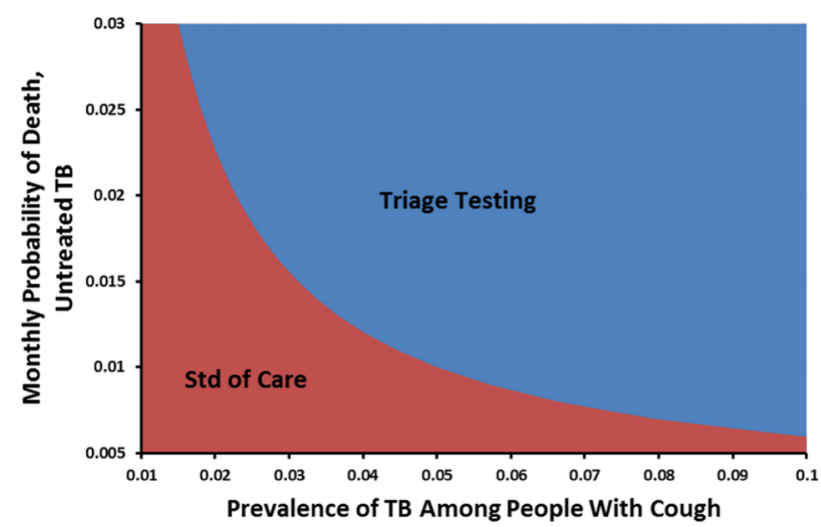

Triage Test Cost $=\$ 3$

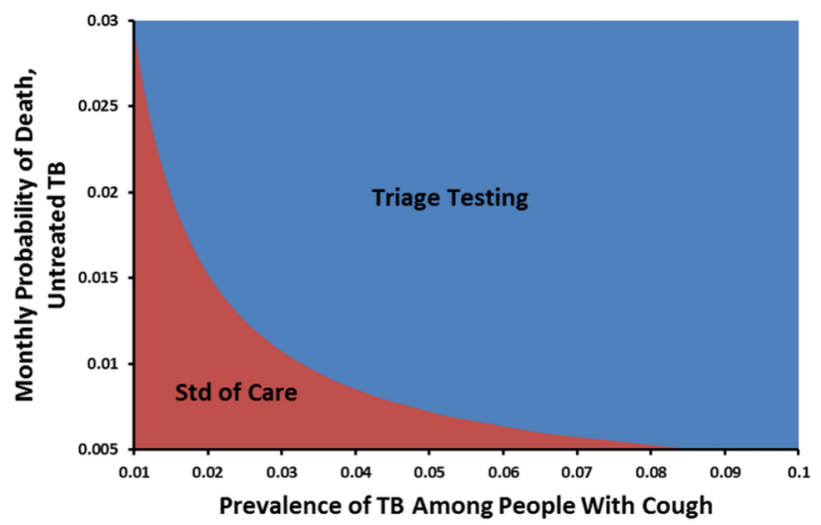

Triage Test Cost $=\$ 10$

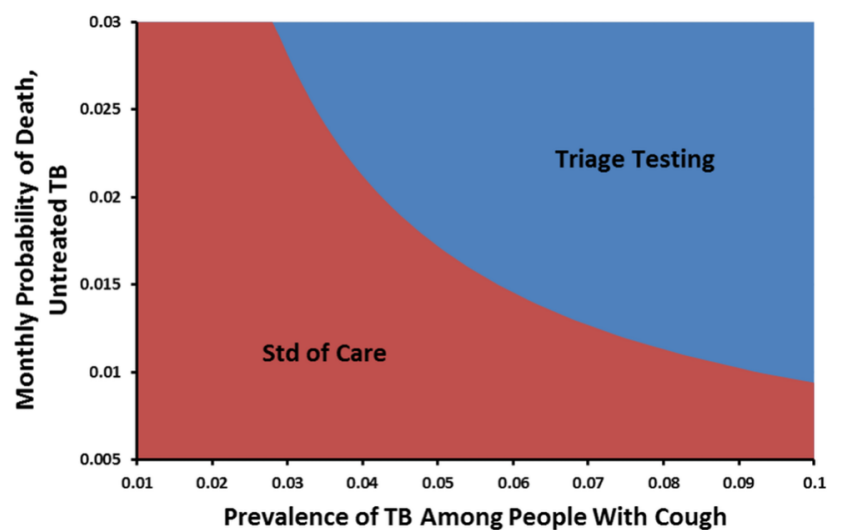

Figure 4 Three-way sensitivity analysis. Blue areas denote combinations of test cost, tuberculosis (TB) prevalence and untreated TB mortality that result in an incremental cost-effectiveness for triage testing, relative to the standard (std) of care, below Uganda's per capita gross national income in 2014 (US\$680) for 1 year of life gained. Red areas denote combinations where the standard of care would still be preferred at this willingness-to-pay threshold.

analysis evaluates a counterbalancing approach, namely of using screening for cough, followed by triage testing, to increase the number of people who could be potentially diagnosed with $\mathrm{TB}$, relative to a baseline in which Xpert testing is performed only among people clinically suspected of having TB. As a result, our findings are naturally less optimistic. Even so, we found that triage testing could be a cost-effective approach to systematic screening if implemented in a high-risk population.

While this study illustrates those conditions in which triage testing can be implemented for systematic screening in cost-effective fashion, several assumptions were necessary. The health facility was assumed to have an infrastructure that is sufficient to support Xpert and CXR/CRP 
diagnosis, which may limit external validity. Generalisability is also limited in settings with high prevalence of drug resistance, where $\mathrm{TB}$ treatment costs are substantially higher and treatment outcomes poorer, making triage testing less cost-effective. If systematic screening were also applied in settings where Xpert was not available on-site, additional costs might be incurred from referring patients or samples, but alternatively, increased effectiveness might be realised from more patients otherwise being missed. In some settings, triage testing may need to be based on symptoms other than cough alone; to the extent that the prevalence of TB and characteristics of the triage test are similar in those populations, our results may still be relevant. We assumed that nearly all patients with a positive triage test would proceed to confirmatory testing on the same visit, also resulting in a potentially inflated effectiveness. We used literature estimates for Xpert's unit cost and sensitivity, both of which may be too high in the setting of systematic screening with declining costs of Xpert implementation; reassuringly, sensitivity analysis around these parameters did not materially influence results. We did not account for transmission from patients whose diagnoses would be missed in the absence of cough screening and triage testing. We also did not incorporate the possibilities that a positive triage test might increase the likelihood of empiric treatment or provide ancillary data (eg, mass on CXR) to suggest an alternative diagnosis. From these perspectives, our results may be biased conservatively (against triage testing). Finally, we compared our estimates of costeffectiveness only to international benchmarks, not to costeffectiveness estimates for other $\mathrm{TB}$ interventions in Uganda. A complete evaluation would formally compare the cost-effectiveness of triage testing against that of other interventions that might be considered for implementation instead, using the same funds.

In summary, we demonstrate here the conditions under which screening for cough, followed by triage testing, is likely to be cost-effective for evaluation of TB among adults presenting to healthcare facilities in high-burden, low-income settings such as Uganda. If triage tests can be delivered with sensitivity, specificity and unit cost reflecting current best estimates for CXR or CRP, they must be implemented in populations with high underlying TB prevalence $(5 \%)$ and risk of death from untreated TB (1\% per month) to be cost-effective according to commonly used benchmarks. Research to develop and validate triage tests with more favourable characteristics should therefore be prioritised to make such testing cost-effective in lower-risk populations. Ultimately, these findings suggest that triage algorithms can help improve TB case detection, but cheaper and more accurate tests may be necessary in order for broader implementation of $\mathrm{TB}$ systematic screening in healthcare facilities to be cost-effective.

\section{Handling editor Sanni Yaya}

Contributors DD and MM prepared the original draft of the manuscript. All authors provided input and comments on successive drafts. All authors read and approved the final draft.
Funding This work was funded by the TB Modelling and Analysis Consortium (TB MAC, Bill and Melinda Gates Foundation, OPP1084276).

Competing interests AC reports involvement in studies funded by NIH and PEPFAR on the utility of $C$ reactive protein as a screening test for tuberculosis among people living with HIV. CD reports employment by FIND.

Provenance and peer review Not commissioned; externally peer reviewed.

Data sharing statement No additional data are available.

Open Access This is an Open Access article distributed in accordance with the Creative Commons Attribution Non Commercial (CC BY-NC 4.0) license, which permits others to distribute, remix, adapt, build upon this work noncommercially, and license their derivative works on different terms, provided the original work is properly cited and the use is non-commercial. See: http:// creativecommons.org/licenses/by-nc/4.0/

\section{REFERENCES}

1. WHO. Global Tuberculosis Report 2013. 2013

2. Pai M, Schito M. Tuberculosis diagnostics in 2015: landscape, priorities, needs, and prospects. J Infect Dis 2015;211(Suppl 2): S21-8.

3. Kik SV, Denkinger CM, Jefferson C, et al. Potential market for novel tuberculosis diagnostics: worth the investment? J Infect Dis 2015;211(Suppl 2):S58-66.

4. Sekandi JN, Dobbin K, Oloya J, et al. Cost-effectiveness analysis of community active case finding and household contact investigation for tuberculosis case detection in urban Africa. PLOS ONE 2015;10: e0117009.

5. Herbert N, George A, Masham of Ilton B, et al. World TB Day 2014: finding the missing 3 million. Lancet 2014;383:1016-18.

6. Zarocostas J. A third of world's tuberculosis cases remain undetected, says WHO. BMJ 2010;341:c6396.

7. Silva DR, Muller AM, Tomasini Kda S, et al. Active case finding of tuberculosis (TB) in an emergency room in a region with high prevalence of TB in Brazil. PLOS ONE 2014;9:e107576.

8. Azman AS, Golub JE, Dowdy DW. How much is tuberculosis screening worth? Estimating the value of active case finding for tuberculosis in South Africa, China, and India. BMC Med 2014;12:216.

9. Churchyard GJ, Stevens WS, Mametja LD, et al. Xpert MTB/RIF versus sputum microscopy as the initial diagnostic test for tuberculosis: a cluster-randomised trial embedded in South African roll-out of Xpert MTB/RIF. Lancet Glob Health 2015;3(8): e450-7.

10. Lorent N, Choun K, Thai S, et al. Community-based active tuberculosis case finding in poor urban settlements of Phnom Penh, Cambodia: a feasible and effective strategy. PLOS ONE 2014;9: e92754.

11. Balcha TT, Skogmar S, Sturegård E, et al. Outcome of tuberculosis treatment in HIV-positive adults diagnosed through active versus passive case-finding. Glob Health Action 2015;8:27048.

12. Kranzer $\mathrm{K}$, Afnan-Holmes $\mathrm{H}$, Tomlin $\mathrm{K}$, et al. The benefits to communities and individuals of screening for active tuberculosis disease: a systematic review. Int $J$ Tuberc Lung Dis 2013;17:432-46.

13. Sekandi JN, Neuhauser D, Smyth K, et al. Active case finding of undetected tuberculosis among chronic coughers in a slum setting in Kampala, Uganda. Int J Tuberc Lung Dis 2009;13:508-13.

14. Pantoja A, Kik SV, Denkinger CM. Costs of novel tuberculosis diagnostics - will countries be able to afford it? J Infect Dis 2015;211 (Suppl 2):S67-77.

15. Denkinger CM, Kik SV, Cirillo DM, et al. Defining the needs for next generation assays for tuberculosis. $J$ Infect Dis 2015;211(Suppl 2): S29-38.

16. García-Basteiro AL, Cobelens F. Triage tests: a new priority for tuberculosis diagnostics. Lancet Respir Med 2015;3:177-8.

17. Gabay C, Kushner I. Acute-phase proteins and other systemic responses to inflammation. N Engl J Med 1999;340:448-54.

18. Engel MF, Paling FP, Hoepelman AI, et al. Evaluating the evidence for the implementation of $\mathrm{C}$-reactive protein measurement in adult patients with suspected lower respiratory tract infection in primary care: a systematic review. Fam Pract 2012;29:383-93.

19. Dowdy DW, O'Brien MA, Bishai D. Cost-effectiveness of novel diagnostic tools for the diagnosis of tuberculosis. Int J Tuberc Lung Dis 2008;12:1021-9. 
20. World Bank Uganda Data. World Bank. 2015. http://data.worldbank. org/country/uganda

21. World Bank Exchange Rates. World Bank. 2015. http://data. worldbank.org/indicator/PA.NUS.FCRF

22. United States Consumer Price Index. Bur Labor Stat. http://www.bls. gov/cpi/

23. Tiemersma EW, van der Werf MJ, Borgdorff MW, et al. Natural history of tuberculosis: duration and fatality of untreated pulmonary tuberculosis in HIV negative patients: a systematic review. PLOS ONE 2011;6:e17601.

24. Cattamanchi A, Huang L, Worodria W, et al. Integrated strategies to optimize sputum smear microscopy: a prospective observational study. Am J Respir Crit Care Med 2011;183:547-51.

25. Castelnuovo B. A review of compliance to anti tuberculosis treatment and risk factors for defaulting treatment in Sub Saharan Africa. Afr Health Sci 2010;10:320-4.

26. Vassall A, van Kampen $\mathrm{S}$, Sohn $\mathrm{H}$, et al. Rapid diagnosis of tuberculosis with the Xpert MTB/RIF assay in high burden countries: a cost-effectiveness analysis. PLOS Med 2011;8:e1001120.

27. Corbett EL, Watt CJ, Walker N, et al. The growing burden of tuberculosis: global trends and interactions with the HIV epidemic. Arch Intern Med 2003;163:1009-21.

28. Wilson D, Badri M, Maartens G. Performance of serum C-reactive protein as a screening test for smear-negative tuberculosis in an ambulatory high HIV prevalence population. PLOS ONE 2011;6: e15248.
29. van't Hoog $\mathrm{AH}$, Langendam MW, Mitchell $\mathrm{E}$, et al. A systematic review of the sensitivity and specificity of symptom- and chest-radiography screening for active pulmonary tuberculosis in HIV-negative persons and persons with unknown HIV status. WHO, 2013.

30. Steingart KR, Schiller I, Horne DJ, et al. Xpert $₫$ MTB/RIF assay for pulmonary tuberculosis and rifampicin resistance in adults. Cochrane Database Syst Rev 2014;(1):CD009593.

31. van Cleeff MR, Kivihya-Ndugga LE, Meme $\mathrm{H}$, et al. The role and performance of chest X-ray for the diagnosis of tuberculosis: a cost-effectiveness analysis in Nairobi, Kenya. BMC Infect Dis 2005;5:111

32. Pande T, Pai M, Khan FA, et al. Use of chest radiography in the 22 highest tuberculosis burden countries. Eur Respir $J$ 2015;46:1816-19.

33. Sempa J, Ssennono M, Kuznik A, et al. Cost-effectiveness of early initiation of first-line combination antiretroviral therapy in Uganda. BMC Public Health 2012;12:736.

34. Van't Hoog AH, Cobelens F, Vassall A, et al. Optimal triage test characteristics to improve the cost-effectiveness of the Xpert MTB/ RIF assay for TB diagnosis: a decision analysis. PLoS ONE 2013;8: e82786.

35. Philipsen $\mathrm{RH}$, Sanchez $\mathrm{Cl}$, Maduskar $\mathrm{P}$, et al. Automated chest-radiography as a triage for Xpert testing in resource-constrained settings: a prospective study of diagnostic accuracy and costs. Sci Rep 2015;5:12215. 